\title{
超高效液相色谱-串联质谱法测定果汁中的展青需素
}

\author{
牛 华, 冯 雷*, 牛之瑞, 珠娜, 祝红昆, 王亚琴 \\ (云南省产品质量监督检验研究院, 云南 昆明 650223)
}

摘要:建立了超高效液相色谱-电喷雾串联三重四极杆质谱( UPLC-ESI-MS/MS) 联用技术分析果汁中展青霉素的 方法。浓缩果汁样品经酶解, 乙酸乙酯提取, Oasis HLB 固相萃取 (SPE) 小柱净化后 (澄清果汁直接进行 SPE 净 化), 以 $\mathrm{C} 18$ 色谱柱为分离柱, 以水和乙腈作为流动相进行梯度洗脱, 电喷雾离子源电离、负离子多反应监测模式质 谱进行定性和定量分析。展青霉素在 $1.0 \sim 500.0 \mu \mathrm{g} / \mathrm{L}$ 质量浓度范围内线性关系良好, 相关系数为 0.999 , 方法的 定量限为 $5.0 \mu \mathrm{g} / \mathrm{kg}$; 加标水平为 $5.0 、 25.0$ 和 $100.0 \mu \mathrm{g} / \mathrm{kg}$ 时, 加标回收率为 $80.6 \% \sim 91.8 \%$, 相对标准偏差为 $1.5 \% \sim 7.3 \%$ 。实验结果表明,该方法简单、灵敏、准确,各项技术指标均满足国内外法规要求, 可用于果汁中展青霉 素的检测。

关键词: 超高效液相色谱-串联质谱; 固相萃取; 展青霉素; 果汁

中图分类号: O658 文献标识码:A 文章编号:1000-8713(2012)09-0957-05

\section{Determination of patulin in juice by ultra performance liquid chromatography-tandem mass spectrometry}

\author{
NIU Hua, FENG Lei * , NIU Zhirui, ZHU Na, ZHU Hongkun, WANG Yaqin \\ (Yunnan Institute of Product Quality Supervision and Inspection, Kunming 650223, China)
}

\begin{abstract}
A method was developed for the simultaneous determination of patulin in juice by solid phase extraction ( SPE)-ultra performance liquid chromatography coupled with electrospray ionization tandem mass spectrometry (UPLC-ESI-MS/MS). The cloudy juice was hydrolyzed by enzyme and extracted by ethyl acetate. The extract of cloudy juice was then enriched and purified by an HLB SPE cartridge. If the juice was clear it was directly treated by the cartridge as above. The separation was performed on a C18 column with the gradient elution of acetonitrile and water. The patulin was detected by MS with electrospray negative ionization $\left(\mathrm{ESI}^{-}\right.$) in the mode of multiple reaction monitoring (MRM). The good linearity was observed in the range of $1.0-500 \mu \mathrm{g} / \mathrm{L}$ with the correlation coefficient of 0.999 and the limit of quantitation of $5.0 \mu \mathrm{g} / \mathrm{kg}$. The recoveries were in the range of $80.6 \%-91.8 \%$ at three spiked levels of $5.0,25.0$ and $100.0 \mu \mathrm{g} / \mathrm{kg}$ with the relative standard deviations of $1.5 \%-7.3 \%$. It was proved to be a simple, reliable and accurate method which can fully meet the requirements of patulin detection in juice samples according to most domestic and international legislations.
\end{abstract}

Key words : ultra performance liquid chromatography-tandem mass spectrometry (UPLC-MS/ MS) ; solid phase extraction (SPE) ; patulin; juice

展青需素又叫棒曲霉素 (patulin), 是由青霉 属、曲霉属和根需属等 10 多种真菌代谢产生的真菌 毒素, 化学名称为 4-羟基- $4 H$-呋 [3,2-c] 吡喃-2 $(6 H)$ - 酮 (4-hydroxy-4H-furo[3,2-c]pyran-2(6H)one, 结构式见图 1 ), 分子式为 $\mathrm{C}_{7} \mathrm{H}_{6} \mathrm{O}_{4}$ 。该物质具
有神经毒性、遗传毒性、致畸和潜在的免疫毒 性 ${ }^{[1,2]}$, 对部分动物的半致死量 $\left(\mathrm{LD}_{50}\right)$ 为 $6 \sim 1000$ $\mathrm{mg} / \mathrm{kg}$ 。自 20 世纪 50 年代开始, 美国、欧洲及中国 等对展青霉素污染状况进行的研究调查显示这种真 菌毒素不仅大量污染粮食、饲料,而且对水果及其制 
品的污染程度相当严重 ${ }^{[3]}$ 。由于展青霉素对人类 可能造成的潜在危害, 世界上许多国家都把苹果产 品中展青霉素的限量值规定为 $50 \mu \mathrm{g} / \mathrm{L}$, 将果汁中 展青霉素的最大残留量规定为 $20 \sim 50 \mu \mathrm{g} / \mathrm{L}^{[2]}$ 。

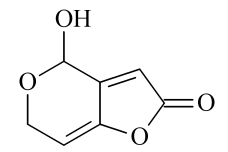

图 1 展青電素的结构式

Fig. 1 Structure of patulin

美国分析化学家协会 (Association of Official Analytical Chemists, AOAC) 规定的展青需素分析 方法为液相色谱法 ${ }^{[4,5]}$ 。我国国家标准方法为改进 的薄层色谱法 (GB/T 5009.185-2003《苹果和山楂 制品中展青霉素的测定》), 该方法的灵敏度都不高 (检出限仅有 $3 \mathrm{mg} / \mathrm{L}$ ) 且操作繁琐。免疫化学法 ${ }^{[6]}$ 可以对展青震素进行快速检测,但此法抗体制备比 较难, 且存在大量的假阳性检测结果, 不能满足准确 检测的要求。目前文献报道较多的展青霉素检测方 法主要有毛细管微乳液电动色谱法 ${ }^{[7]}$ 、气相色谱 法 $^{[8]}$ 、液相色谱法 ${ }^{[9,10]}$ 、气相色谱-质谱法 ${ }^{[11,12]}$ 以及 液相色谱-质谱法 ${ }^{[13-15]}$ 等, 以液相色谱-质谱法居 多,该方法检出限可以达到 $\leqslant 10 \mu \mathrm{g} / \mathrm{L}$, 甚至 $4 \sim 5$ $\mu \mathrm{g} / \mathrm{L}_{\circ}$ 展青霉素的实际检测应用中以液相色谱法 居多, 前处理过程相对于气相色谱法较简单, 不需要 进行衍生化, 但液相色谱法存在灵敏度不高, 且容易 出现假阳性结果的弊端, 因此, 可以准确定性及定量 的液相色谱-质谱法越来越受到人们的重视。液相 色谱-质谱法分析展青霉素的样品前处理方法主要 有液-液萃取法 ${ }^{[16]}$ 、固相萃取法 ${ }^{[17]}$ 、基质固相分散 法 ${ }^{[18]}$ 等。我们在前人研究的基础上, 比较了 $\mathrm{C} 18$ 固相萃取法和展青霉素多功能净化柱 (MycoSep 228 AflaPat)净化等前处理方法, 并建立了果汁中 展青霉素的超高效液相色谱-串联质谱 (UPLC-MS/ MS) 检测方法。

\section{1 实验部分}

\section{1 仪器、试剂与材料}

Acquity 超高效液相色谱仪(美国 Waters 公 司), API 3200 三重四极杆串联质谱联用仪 (美国 $\mathrm{AB}$ 公司);Analyst 1.5 质谱工作站 (Build 4019, 美 国 $\mathrm{AB}$ 公司)。GL-12B 高速离心机(上海安亭科学 仪器厂), N-EVAP 氮吹仪 (美国 Organomation 公 司), CPA225D 电子天平 (德国 Sartorius 公司),20 孔固相萃取装置(美国 Waters 公司)。
展青霉素标准品 (纯度 99\%, 美国 Sigma 公 司), 乙腈、甲醇、乙酸乙酯 (色谱纯, 美国 Fisher 公 司), $18.2 \mathrm{M} \Omega \cdot \mathrm{cm}$ 超纯水由赛多利斯超纯水机生 产, 果汁样品购买于昆明市场。Oasis HLB 固相萃 取小柱 (3 mL/60 mg, 美国 Waters 公司), SepPak $\mathrm{C} 18$ 固相萃取小柱 $(6 \mathrm{~mL} / 500 \mathrm{mg}$, 美国 Waters 公 司), MycoSep 228 AflaPat 多功能净化柱 (配 10 $\mathrm{mL}$ 样品净化试管, 美国 Romer 公司)。果胶酶 (美 国 Sigma 公司)。

\section{2 标准溶液及缓冲液的配制}

称取适量展青需素标准品, 用乙酸乙酯配制成 $1000 \mathrm{mg} / \mathrm{L}$ 的标准储备溶液, 避光保存于 $-20{ }^{\circ} \mathrm{C}$ 冰 箱中。准确移取适量的展青霉素标准储备溶液, 用 氮气吹干后, 用 $0.2 \%(\mathrm{v} / \mathrm{v})$ 乙酸配成 $2 \mathrm{mg} / \mathrm{L}$ 的标 准中间溶液, 避光于 $4{ }^{\circ} \mathrm{C}$ 冰箱中。使用时, 以初始流 动相稀释成 $1.0 、 5.0 、 10.0 、 50.0 、 100.0 、 500.0$ $\mu \mathrm{g} / \mathrm{L}$ 系列标准溶液供 UPLC-MS/MS 测定。

\section{3 样品前处理}

\subsection{1 酶解与萃取}

对于浓缩果汁或者混浊样品, 取 $4 \mathrm{~g}$ 试样 (精确 到 $0.01 \mathrm{~g}$ ) 于一具塞试管中, 加人 $20 \mathrm{~mL}$ 水与 200 $\mu \mathrm{L}$ 果胶酶混匀, 室温下避光过夜, 在酶解后的溶液 中加人 $20 \mathrm{~mL}$ 乙酸乙酯, 浴旋提取 $3 \mathrm{~min}$, 于 4000 $\mathrm{r} / \mathrm{min}$ 离心 $5 \mathrm{~min}$, 转移上层乙酸乙酯提取液, 再用 $20 \mathrm{~mL}$ 乙酸乙酯重复提取一次。合并两次乙酸乙酯 提取液, 过无水硫酸钠后, 取 $20 \mathrm{~mL}$ 氮吹浓缩至干, 用 $2 \mathrm{~mL} 0.2 \%(\mathrm{v} / \mathrm{v})$ 乙酸溶液溶解残渣并定容。

对于澄清果汁样品, 取 $2 \mathrm{~g}$ 试样 (精确到 0.01 g) 直接进行净化。

\subsection{2 净化}

Oasis HLB 和 SepPak C18 固相萃取小柱操作 方法为: 依次加人 $3 \mathrm{~mL}$ 甲醇和 $3 \mathrm{~mL}$ 水活化。将上 述试样上样于活化后的固相萃取柱上,用 $2 \mathrm{~mL} 1 \%$ (v/v) 乙酸溶液淋洗, 流速控制在 $3 \mathrm{~mL} / \mathrm{min}$ 左右, 抽真空 $3 \sim 5 \mathrm{~min}$, 然后加人 $5 \mathrm{~mL}$ 乙酸乙酯溶液洗 脱, 收集洗脱液于氮气 $40{ }^{\circ} \mathrm{C}$ 下吹至近干, 准确加人 $0.5 \mathrm{~mL} 0.2 \%(\mathrm{v} / \mathrm{v})$ 乙酸溶液, 浴旋混匀 $30 \mathrm{~s}$, 过 $0.45 \mu \mathrm{m}$ 滤膜后,供 UPLC-MS/MS 测定。

MycoSep 228 AflaPat 固相萃取小柱操作方法 为: 取 $2 \mathrm{~mL}$ 酶解萃取后的试样或澄清果汁样品于 样品净化试管中, 加人 $6 \mathrm{~mL}$ 乙腈, 将 MycoSep 228 净化柱放于试管正上方, 手轻轻向下推柱体,使液面 上升速度保持在 $1.0 \mathrm{~mL} / \mathrm{min}$ 左右, 待样液全部通 过柱体后, 准确移取 $4 \mathrm{~mL}$ 上层净化液于 $40{ }^{\circ} \mathrm{C}$ 下用 氮吹浓缩至近干。加人 $0.5 \mathrm{~mL} 0.2 \%(\mathrm{v} / \mathrm{v})$ 乙酸溶 
液, 混匀, 过 $0.45 \mu \mathrm{m}$ 滤膜后, 供 UPLC-MS/MS 测定。

\section{4 色谱-质谱条件}

\section{4 .1 色谱条件}

色谱柱为美国 Waters 公司 Acquity UPLC $\mathrm{BEH} \mathrm{C} 18$ 柱 $(100 \mathrm{~mm} \times 2.1 \mathrm{~mm}, 1.7 \mu \mathrm{m})$ 。流动 相 $\mathrm{A}$ 为水, 流动相 $\mathrm{B}$ 为乙腈, 流动相梯度: $0 \sim 1$ $\min , 10 \% \mathrm{~B} ; 1 \sim 3 \min , 10 \% \mathrm{~B} \sim 60 \% \mathrm{~B} ; 3 \sim 4$ $\min , 60 \% \mathrm{~B}$; $4 \sim 4.1 \mathrm{~min}, 60 \% \mathrm{~B} \sim 10 \% \mathrm{~B} ; 4.1 \sim 6$

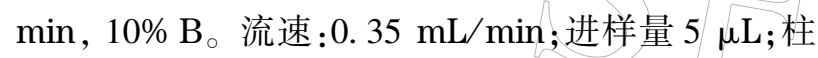
温: $30{ }^{\circ} \mathrm{C}$ 。

\subsection{2 质谱条件}

扫描方式: 电喷雾负离子模式 $\left(\mathrm{ESI}^{-}\right)$; 检测方 式:多反应监测 $(\mathrm{MRM})$; 喷雾电压: $-4500 \mathrm{~V}$; 气帘 气: $69 \mathrm{kPa}$; 碰撞气: $34.5 \mathrm{kPa}$; 去溶剂温度: $600{ }^{\circ} \mathrm{C}$; 雾化气: $345 \mathrm{kPa}$; 辅助气: $345 \mathrm{kPa}$ 。定量离子对 $(m / z: 152.9 / 108.9)$ 和定性离子对 $(m / z: 152.9 /$ $81.0)$ 的扫描时间均为 $100 \mathrm{~ms}$; 定量离子对的去簇 电压 (DP) 为 $-20 \mathrm{~V}$, 碰撞能量 $(\mathrm{CE})$ 为 $-13 \mathrm{eV}$; 定 性离子对的 $\mathrm{DP}$ 为 $-20 \mathrm{~V}, \mathrm{CE}$ 为 $-20 \mathrm{eV}$ 。

\section{2 结果与讨论}

\section{1 质谱检测及色谱分离条件的优化}

质谱检测采用 $2 \mathrm{mg} / \mathrm{L}$ 标准溶液以针洜直接进 样的方式分别在正、负离子模式下进行全扫描, 以确 定展青霉素的准分子离子, 然后以准分子离子作为 母离子, 对其子离子进行全扫描。在本研究条件下, 由于展青震素具有很强的电负性, 其负离子模式下 的准分子离子峰强度为 $1.7 \times 10^{7}$, 高于正离子模式 下的准分子离子峰强度, 所以在负离子模式下的灵 敏度比正离子模式下高。负离子模式下的电喷雾离 子化使展青霉素产生 $m / z 152.9$ 的准分子离子峰 $[\mathrm{M}-\mathrm{H}]^{-}$, 对碰撞能量、去簇电压等质谱条件优化 后得到子离子 $m / z 108.9$ 和 $m / z 81.0$, 选取干扰 小、丰度强的离子对 $m / z 152.9 / 108.9$ 作为定量离 子对, 展青霉素的二级质谱图见图 2 , 优化后的质谱 参数见 1.4 .2 节。

展青霉素属于低相对分子质量、高极性的化合 物,在色谱分离过程中比较了 BEH C18 和 Acquity HILLIC 柱 $(100 \mathrm{~mm} \times 2.1 \mathrm{~mm}, 1.7 \mu \mathrm{m}$, Waters 公 司)两种不同类型的色谱柱。展青霉素在 BEH C18 柱上的保留时间为 $1.7 \mathrm{~min}$, 峰宽为 $0.1 \mathrm{~min}$; 在 HILLIC 柱上的保留时间为 $2.3 \mathrm{~min}$, 峰宽为 0.3 $\min$ 。展青霉素在反相材料 C18 的色谱柱上虽然保 留时间较短,但可以和干扰物质完全分离且峰形较

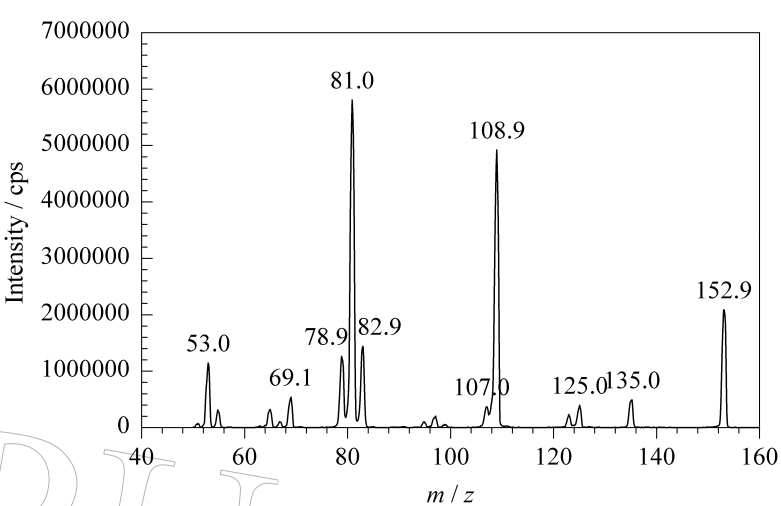

图 2 展青霉素的二级质谱图

Fig. $2 \mathrm{MS}^{2}$ spectrum of patulin

好; 而在 HILLIC 色谱柱上保留时间虽长, 但峰形较 差而影响检测的灵敏度, 且 HILLIC 色谱柱要求流 动相中水相一般不超过 50\% (v/v)、样品溶剂必须 以有机相为主, 不能满足样品前处理时展青霉素的 提取要求。因此,最终选择使用 BEH C18 色谱柱对 展青霉素进行分离。

本实验同时考察了不同流动相体系 (包括水-乙 腈 $(9: 1, \mathrm{v} / \mathrm{v}) 、 0.2 \%(\mathrm{v} / \mathrm{v})$ 乙酸水溶液-乙腈 $(9: 1$, $\mathrm{v} / \mathrm{v}) 、 10 \mathrm{mmol} / \mathrm{L}$ 乙酸铵溶液-乙腈 $(9: 1, \mathrm{v} / \mathrm{v})$ 等 $)$ 对展青霉素色谱响应的影响。在上述 3 种流动相体 系下分别对 $50 \mu \mathrm{g} / \mathrm{L}$ 的标准品进行检测, 展青震素 色谱峰的信噪比分别为 132,110 和 95 , 说明采用 水-乙腈作为流动相体系时展青霉素的响应最强, 同 时考虑到展青霉素在酸性溶液中较为稳定, 因此采 用上述水-乙腈溶液作为流动相, 以 $0.2 \%(\mathrm{v} / \mathrm{v})$ 乙 酸水溶液作为样品定容溶液。

\section{2 固相萃取小柱的选择}

本文比较了常用于真菌毒素样品净化的几种固 相萃取柱: Oasis HLB 柱, SepPak C18 柱和 MycoSep 228 AflaPat 多功能净化柱, 通过进行加标回 收试验考察固相萃取小柱对样品净化效果的影响, 加标水平、回收率及重现性结果见表 1 。从表 1 可 以看出, SepPak C18 柱的回收率和重现性最差, 达 不到检测需求。这是由于展青霉素在 $\mathrm{C} 18$ 小柱硅 胶基的硅烷化填料上保留时间很短, 极易被洗脱, 所 以整个净化过程也较难控制。而 Oasis HLB 柱和 MycoSep 228 AflaPat 多功能净化柱的回收率和重 现性均能满足实验要求。Oasis HLB 和 MycoSep 228 AflaPat 采用的填料均能够很好地对样品进行 净化, 保证了样品的回收率。但相比较而言, MycoSep 228 AflaPat 多功能净化柱价格相对较高, 且 操作方法较为复杂; 而 Oasis HLB 柱相对价格低廉, 操作简便, 适合批量检测。因此本文选择 Oasis 
HLB 柱用于果汁中展青霉素的检测工作。

表 1 展青霉素在不同固相萃取柱上的回收率和精密度 ( 以相对标准偏差 (RSD) 计) $(n=6)$

Table 1 Recoveries and precisions (relative standard deviations (RSDs) ) of patulin purified with different SPE cartridges $(n=6)$

\begin{tabular}{lcccc}
\hline SPE cartridge & $\begin{array}{c}\text { Added } \\
(\mu \mathrm{g} / \mathrm{kg})\end{array}$ & $\begin{array}{c}\text { Found/ } \\
(\mu \mathrm{g} / \mathrm{kg})\end{array}$ & $\begin{array}{c}\text { Recovery/ } \\
\%\end{array}$ & $\begin{array}{c}\text { RSD/ } \\
\%\end{array}$ \\
\hline Oasis HLB & 12.5 & 11.2 & 89.6 & 7.4 \\
& 25.0 & 23.1 & 92.4 & 7.1 \\
SepPak C18 & 50.0 & 47.2 & 94.4 & 6.8 \\
& 12.5 & 6.8 & 54.4 & 9.7 \\
& 25.0 & 16.7 & 68.8 & 8.6 \\
MycoSep 228 & 50.0 & 38.6 & 77.2 & 11.2 \\
AflaPat & 12.5 & 11.6 & 92.8 & 5.9 \\
& 25.0 & 24.7 & 98.8 & 6.7 \\
& 50.0 & 51.1 & 102.2 & 6.5 \\
\hline
\end{tabular}

\section{3 线性范围和定量限}

配制了浓缩果汁的基质标准溶液、澄清果汁的 基质标准溶液和无基质的标准溶液, 以峰面积 $(Y)$ 对质量浓度 $(X, \mu \mathrm{g} / \mathrm{L})$ 进行线性回归, 回归方程依 次为: $Y=446 X+286(r=0.997), Y=440 X+457(r$ $=0.999), Y=452 X-106(r=0.999)$, 从标准曲线 的斜率可以看出基质对定量的影响较小, 考虑到实 验的简便快捷,最终采用无基质标准溶液制作工作 曲线。结果表明展青霉素在 $1.0 \sim 500.0 \mu \mathrm{g} / \mathrm{L}$ 时, 相关系数为 0.999 ,相关系数良好。在空白浓缩果 汁中添加展青霉素标准品, 当添加水平为 5.0 $\mu \mathrm{g} / \mathrm{kg}$ 时, 信噪比 $(S / N)$ 远远大于 10 , 而此含量远 低于标准限量值 $50 \mu \mathrm{g} / \mathrm{kg}$ ( GB 2761-2005《食品中 真菌毒素限量》), 故确定其定量限为 $5.0 \mu \mathrm{g} / \mathrm{kg}$ 。 $50.0 \mu \mathrm{g} / \mathrm{L}$ 展青需素标准溶液的总离子流色谱 ( TIC) 图见图 3。

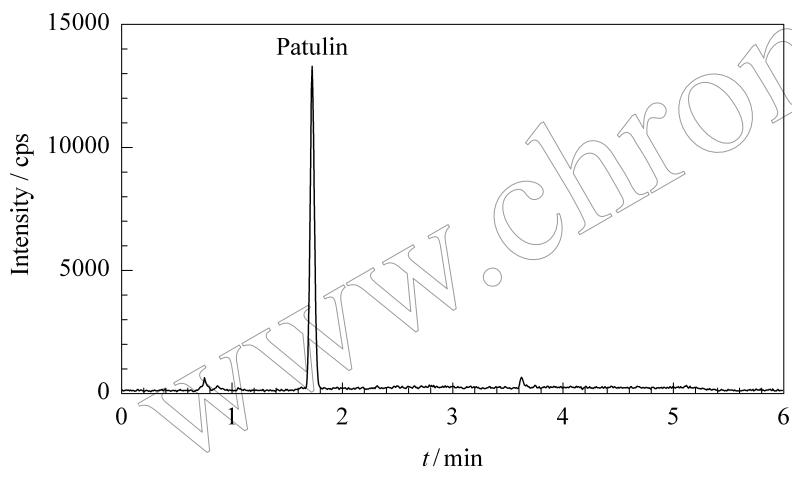

图 $350.0 \mu \mathrm{g} / \mathrm{L}$ 展青霉素标准溶液的 TIC 图

Fig. 3 Total ion chromatogram (TIC) of patulin standard solution at $50.0 \mu \mathrm{g} / \mathrm{L}$

Mobile phases: water (A), acetonitrile (B). Gradient elution: $0-1 \min , 10 \% \mathrm{~B}$; $1-3 \min , 10 \% \mathrm{~B}-60 \% \mathrm{~B} ; 3-4 \min$, $60 \%$ B; 4 - 4.1 min, $60 \%$ B - 10\% B; $4.1-6$ min, 10\% B. Flow rate: $0.35 \mathrm{~mL} / \mathrm{min}$. Injection volume: $5 \mu \mathrm{L}$. Column temperature: $30{ }^{\circ} \mathrm{C}$.

\section{4 回收率和精密度}

称取 $4 \mathrm{~g}$ 浓缩果汁空白试样, 添加标准溶液, 使 加标水平分别达到 $5.0,25.0$ 和 $100.0 \mu \mathrm{g} / \mathrm{kg}$, 充分 混匀, 按照本文建立的方法进行测定, 每个添加水平 作 6 份平行测定。方法的回收率为 80.6\% $91.8 \%$, 相对标准偏差 (RSD) 为 $1.5 \% \sim 7.3 \%$ (见表 2 ), 说明本方法的回收率好,精密度高。

表 2 方法的回收率和精密度试验结果 $(n=6)$

Table 2 Results of recovery and precision test $(n=6)$

\begin{tabular}{rccc}
\hline Added $/(\mu \mathrm{g} / \mathrm{kg})$ & Found $/(\mu \mathrm{g} / \mathrm{kg})$ & Recovery $/ \%$ & $\mathrm{RSD} / \%$ \\
\hline 5.0 & 4.0 & 80.6 & 7.3 \\
25.0 & 21.7 & 86.9 & 5.8 \\
100.0 & 91.8 & 91.8 & 1.5 \\
\hline
\end{tabular}

\section{5 实际样品检测}

我们采用本方法对市场上 10 种品牌的浓缩果 汁及澄清果汁进行了检测, 在其中一个品牌的浓缩 苹果汁中检测出了展青霉素, 检测值为 $8.9 \mu \mathrm{g} / \mathrm{kg}$, 其他样品中均未检出展青霉素。阳性样品的 TIC 图见图 4。

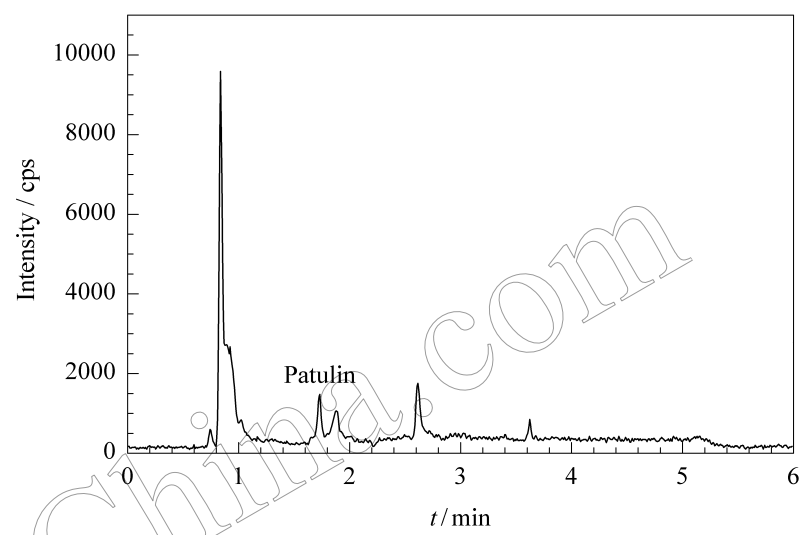

图 4 阳性样品中展青霉素的 TIC 图

Fig. 4 TIC of patulin in a positive sample Conditions were the same as in Fig. 3.

从图 4 中可以看出, 目标物展青需素与干扰物 质达到了基线分离,方法的净化效果较好。

\section{3 结论}

本文经过样品处理固相萃取小柱的选择、洗脱 条件、色谱分离及质谱检测条件的优化, 建立了 UPLC-MS/MS 检测果汁中展青需素的方法。样品经 提取后过 Oasis HLB 固相萃取小柱净化, 采用 BEH C18 色谱柱分离, UPLC-MS/MS 方法进行检测。该 方法前处理操作简便,净化效果好, 回收率和精密度 高, 分析周期短, 能够满足于我国果汁样品中对于展 青需素的测定要求。 


\section{参考文献：}

[1] Barkai-Golan R, Paster N. Mycotoxins in Fruits and Vegetables. San Diego, CA: Elsevier, 2008

[2] Yang X Q, Li J K, Guo D Z, et al. Chinese Journal of Food Hygiene (杨晓强, 李家奎, 郭定宗, 等. 中国食品卫生杂 志), 2007, 19(2) : 165

[3] Yu S R, Liu J. Foreign Medical Sciences: Section of Hygiene (俞世荣, 刘江. 国外医学: 卫生学分册), 1996, 23 (1) : 40

[4] AOAC Official Method 995.10. [2012-3-16 ]. http: // down. 40777. cn/stardard/8/49. 7. 02\%20AOAC\% 20Official\% 20Method\%20995. 10\%20Patulin\%20in\%20Apple\%20Juice. pdf

[5] AOAC Official Method 2000. 02. [2012-3-16]. http://fjc100. cn/gzwmanage/UploadFiles/2008826101225339. pdf

[6] De Champdoré M, Bazzicalupo P, De Napoli L, et al. Anal Chem, 2007, 79(2): 751

[7] Murillo-Arbizu M, González-Penas E, Hansen S H, et al. Food Chem Toxicol, 2008, 46(6) : 2251

[8] Collin S, Bodart E, Badot C, et al. J I Brewing, 2008, 114 (2) $: 167$

[9] Baert K, De Meulenaer B, Kasase C, et al. Food Chem, $2007,100: 1278$
[10] Qiu N X, Niu P F, Su X J. Food Science (仇农学, 牛鹏飞, 苏肖洁. 食品科学) , 2008, 29(6): 312

[11] Zhou J N, Wu P G. Chinese Journal of Health Laboratory Technology (周继恩, 吴平谷. 中国卫生检验杂志), 2010, 20(12) : 3237

[12] Li F G, Yao W Q, Tian Y H, et al. Chinese Journal of Chromatography (李锋格, 姚伟琴, 田延河, 等. 色谱), 2010, 28(7): 720

[13 ] Gong X M, Ren Y P, Dong J, et al. Journal of Instrumental Analysis (宫小明, 任一平, 董静, 等. 分析测试学报), $2011,30(1): 6$

[14] Yang J Z, Wei W, Zhu W X, et al. Food Science (杨冀州, 魏蔚, 祝伟霞, 等. 食品科学), 2009, 30(4): 162

[15] Kataoka H, Itano M, Ishizaki A, et al. J Chromatogr A, $2009,1216(18): 3746$

[16] Yin J Y, Ni M L, Xie D H, et al. Journal of Analytical Science (殷居易, 倪梅林, 谢东华, 等. 分析科学学报), 2007, $23(1): 119$

[17] Li J K, Wu R N, Hu Q H, et al. Food Control, 2007, 18 : 530

[18] Wu R N, Dang Y L, Niu L, et al. J Food Compos Anal, 2008, $21: 582$ 